\title{
Online Fault Detection Approach of Unpredictable Inputs: Application to Handwriting System
}

\author{
Ines Chihi (i) and Mohamed Benrejeb \\ Laboratory of Research in Automation (LA.R.A), National School of Engineering Tunis (ENIT), \\ Campus Universitaire El Manar 1 BP 37, Tunisia \\ Correspondence should be addressed to Ines Chihi; chihi4ines@hotmail.fr
}

Received 24 August 2018; Accepted 15 November 2018; Published 3 December 2018

Academic Editor: Lingzhong Guo

Copyright (C) 2018 Ines Chihi and Mohamed Benrejeb. This is an open access article distributed under the Creative Commons Attribution License, which permits unrestricted use, distribution, and reproduction in any medium, provided the original work is properly cited.

\begin{abstract}
Many investigators are interested in improving the control strategies of hand prosthesis to make it functional and more convenient to use. The most used control approach is based on the forearm muscles activities, named 'ElectroMyoGraphic' (EMG) signal. However, these biological signals are very sensitive to many disturbances and are generally unpredictable in time, type, and level. This leads to inaccurate identification of user intent and threatens the prosthesis control reliability. This paper proposed a realtime fault detection and localization approach applied to handwriting device on the plane. This approach allows connecting inputs (IEMG signals)/outputs (pen tip coordinates) data as a parametric model for Multi-Inputs Multi-Outputs (MIMO) system. The proposed approach is considered as a model-independent abrupt or intermittent fault detection method and as an alternative solution to the unpredictable input observer based techniques, without any observability requirements. This approach allows detecting, in real time, several types of faults in one or two inputs signals and in the same or different instants. Our study is appropriate for many rapidly expanding fields and practices, including biomedical engineering, robotics, and biofeedback therapy or even military applications.
\end{abstract}

\section{Introduction}

In the last decades, robot control is considered as an important research field, especially for robots intervening in the tasks of everyday life (assistance robots, social robots, service robots, clinical application robots, etc.). Many investigators are interested in improving the quality of prosthesis to make it functional and more convenient to use. The increase in functionality is mainly based on the progression of the control strategies. The most used control approach is based on the amplifier electrical activity of the muscles, named electromyographic signal (EMG), which allows directly encoding the orders generated by the brain [1-4].

The wealth of information of these biological signals leads several researches to propose approaches based on the muscular activities control. For example, in [7] EMG signals of ten muscles were used to control an artificial hand with four fingers. Schulz et al. proposed, in [8], an artificial hand with hydraulically driven multifunction. In this context, different reviews of controlling by electrical muscles activities are proposed in [9-12].

However, EMGs are very sensitive to many disturbances in EMG recordings and are generally unpredictable in time, type, and level. The characteristics of the muscles activities are easily affected by many factors, such as recording over layers of muscles, fat, and tissue, abrupt changing of the electrodes positions, sweat of the prosthesis wearer at the recording site, changes of the impedance of the electrode, filtering method, noise of measure, disturbances, and user fatigue [13-15]. All these conditions lead to inaccurate identification of user intent and threaten the prosthesis control reliability [16-20].

In order to solve these problems, software integrated electromyographic (IEMG) sensor and intelligent techniques are used, in [21], to replace physical sensors. It was also used as a part of fault detection approaches, where the output is compared to the corresponding sensor.

In [22], Zhang et al. proposed a practical fault-tolerant module for robust EMG, based on Mahalanobis distance 
analysis [23]. Using the Zhang fault approach, Resnik included in [24] a fault detection module which detects faults in the input signals (EMG). In 2018, Huang proposed an EMG fault detector, taking into account only 3 kinds of signal faults (e.g., EMG signal drift and saturation, additional noise, and variation of EMG magnitude) [25].

In fact, nowadays, Fault Detection and Diagnosis (FDD) have been growing interest generated in several fields, especially in the emerging field of bio-robotics. For these reasons, this topic has been addressed in many previous works. In this sense, a bibliographic review on reconfigurable faulttolerant control approach is presented in [26]. In [27], a data mapping fault detection approach was proposed to detect actuator faults of a manipulator robot. Indeed, FDD can be typically classified into two different classes: model-based and model-free approaches. For model-based class, different methods using mathematical models have been developed in [27-32]. Furthermore, approaches that are not based on system modeling used generally intelligent methods like neural network or fuzzy logic concepts. In several works, deep learning have been developed to compute the residuals and to detect sensor or actuator faults for different types of systems (linear, nonlinear electrical, hydraulic, etc.), as presented in $[33,34]$.

The main contribution of this paper is the proposal of a real-time fault detection and localization approach applied to handwriting device allowing producing cursive letters and geometric shapes from electromyographic signals of only two forearm muscles. As already mentioned, these signals are subject to uncertainties and internal/external disturbances.

In this sense, a predictive model is particularly challenging for the estimation of faulty unpredictable inputs. This model allows connecting inputs (IEMG signals)/outputs (pen tip coordinates) data as a parametric model for MultiInputs Multi-Outputs (MIMO) system. Afterward, Recursive Least Squares (RLS) algorithm is used to identify the model's parameters. Our focus, hereafter, will be the use of normalized residue to detect in real-time different kinds of actuator fault arising on the studied device.

Unlike previous fault detection studies, developed for myoelectric prosthesis and devices ensuring simple movement (opening, closing), the developed approach is proposed for handwriting system allowing us to generate cursive and complex pattern, especially cursive Arabic letters,.$h$, " HA» and $s$, «SIN », which are composed by combined movements, vertical, horizontal, oblique, etc.

On the other hand, our study is considered as a model-independent abrupt or intermittent fault detection method and as an alternative solution to the unpredictable input observer based techniques, without any observability requirements.

Indeed, the unpredictable characteristics and the variation of the biological parameters, related to the handwriting process, like muscle mass and muscle fatigue, which interfere with physical and psychical variation of scripters, make the task of modeling complex and tedious. Therefore, free models approaches are preferable in this case. The proposed fault detection method is part of this category of model.
In addition, the developed fault detection approach allows detecting several types of faults in one or two inputs signals and in the same or different instants. These faults may be due to technical problems (defective electrode), physical (sweat), or even damage to the components that constitute the system to be supervised.

The present paper is organized as follows: after presentation of the handwriting experimental approach in the second section, the third section focuses on the study and classification of different kinds of faults that can be detected during the handwriting act. The fourth section shows the development of actuator online fault modeling approach using damaged outputs to detect faulty inputs.

\section{Experimental Approach}

Handwriting movement, on the plane $(x, y)$, is considered as a complex movement based on two electromyography signals, EMG1 and EMG2, of the most active forearm muscles, namely, the "Abductor Pollicis Longus" and the "Extensor Capri Ulnaris" [5, 35]. The first muscle is responsible for the vertical displacement and the second one for the horizontal motions.

In this sense, an experimental approach was proposed, in Hiroshima City University, to record at the same instant cursive Arabic letters or geometric forms and two forearm EMG signals [35].

In fact, the measuring data were synchronized by sending a step signal from the parallel interface port on the computer to the data recorder. This experimentation had required the following equipment:

(i) Digital table of the brand "WACOM, KT-0405-RN".

(ii) Preamplifiers "TEAC, AR-C2EMG1".

(iii) Data recorder "TEAC, DR-C2".

(iv) Bipolar surface electrodes (MEDICOTEST, Blue Sensor N-00-S).

(v) Computer.

Figure 1 indicates the positioning of electrodes on the writer's arm. Electrodes, indicated by "chl", are relative to the first muscle and those relative to the second muscle are indicated by "ch2" [5].

In Figure 2, the recorded data for the Arabic letter, .h, "HA" are presented [35].

However, it is difficult to get the useful information from muscles activities. Therefore, a variety of signal processing techniques are used to make EMG waveforms easier to interpret. Indeed, the fluctuation of EMG's magnitudes can be filtered to obtain new curves called integrated EMG (IEMG), represented by dotted red curves in Figure 2(b) [35].

We note IEMG1 and IEMG2, the integrated EMG1 and EMG2 signals, respectively.

The studied system is sensitive to different types of faults due to many problems: electronic, mathematic, biologic, etc. Electromyography signals are directly related to several morphological and technical properties that influence the quality of these signals (filtering method, noise and disturbances, 


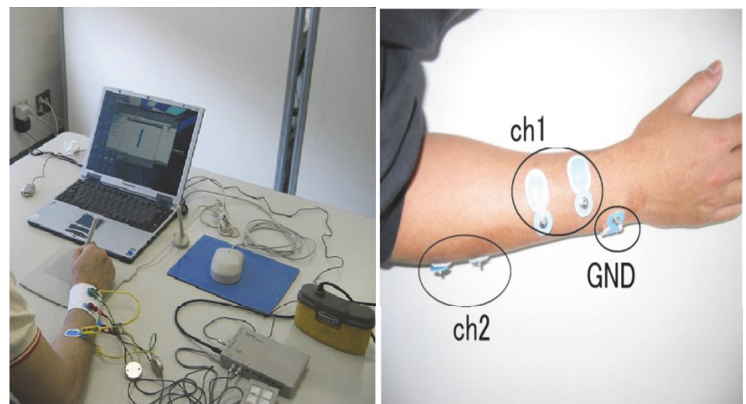

FIGURE 1: Experimental assembly and electrodes' positions on the writer's arm [5].
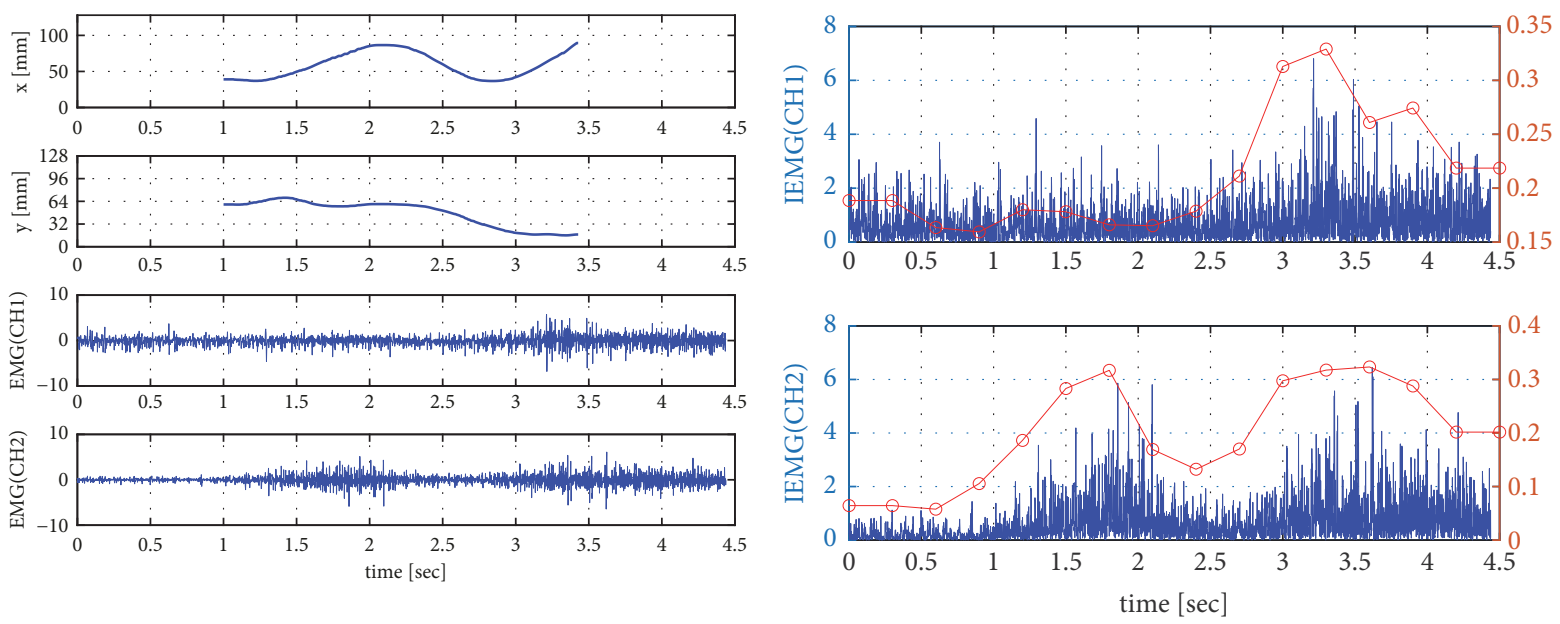

(a)

(b)

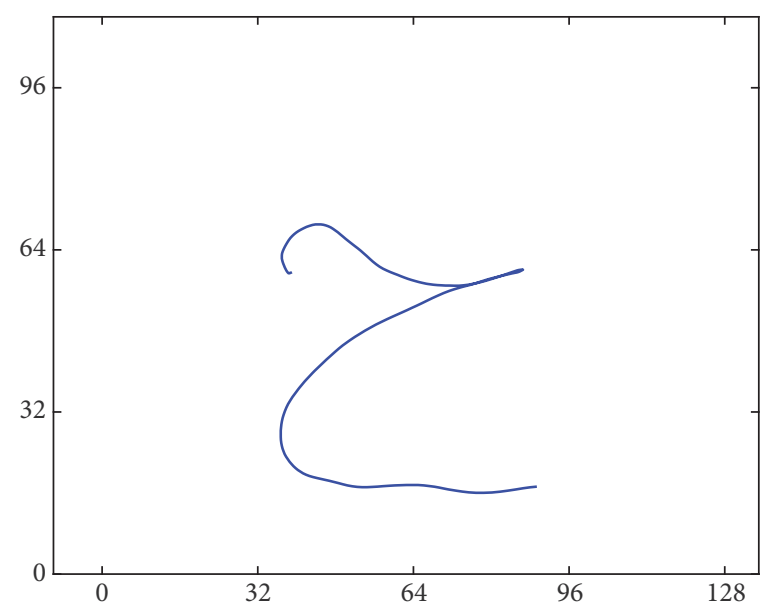

(c)

FIGURE 2: The letter, 乙, «HA». (a) Movement on the plane $(x, y)$ and EMG signals. (b) IEMG signals. (c) Form.

distance between the muscles and the electrodes that are the sensors of the muscular activities, etc.).

An undesirable deviation could occur on the written shape if a fault appears on the handwriting process inputs. To conclude, the fault that damages EMG signals affects outputs of the handwriting model, and this will further make the studied system reconstructing faulty graphic traces.
Figure 3 shows the impact of faulty inputs on the quality of writing. Its presents some faulty responses of two cursives Arabic letters, .h, "HA" in Figure 3(a), s, "SIN" in Figure 3(b). The experimental recorded data, in fault free case, are represented by discontinuous lines. The model's outputs, responses for faulty inputs, are presented by a continuous line [6].

However, electromyography signal is considered as a complex signal with the particularity of variation according 


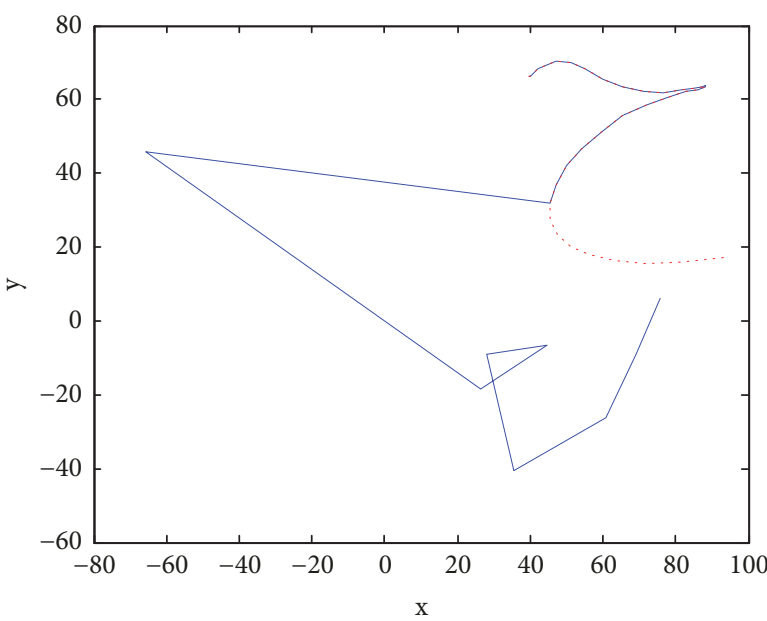

(a)

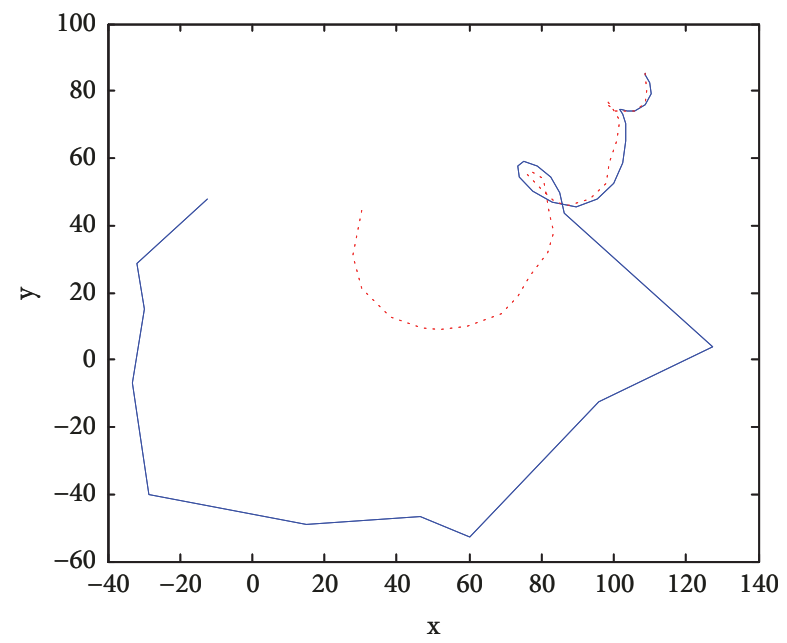

(b)

FIGURE 3: Damaged Arabic letters due to faulty inputs. (a) Arabic letter, 乙, «HA». (b) Arabic letter, س, «SIN » [6].

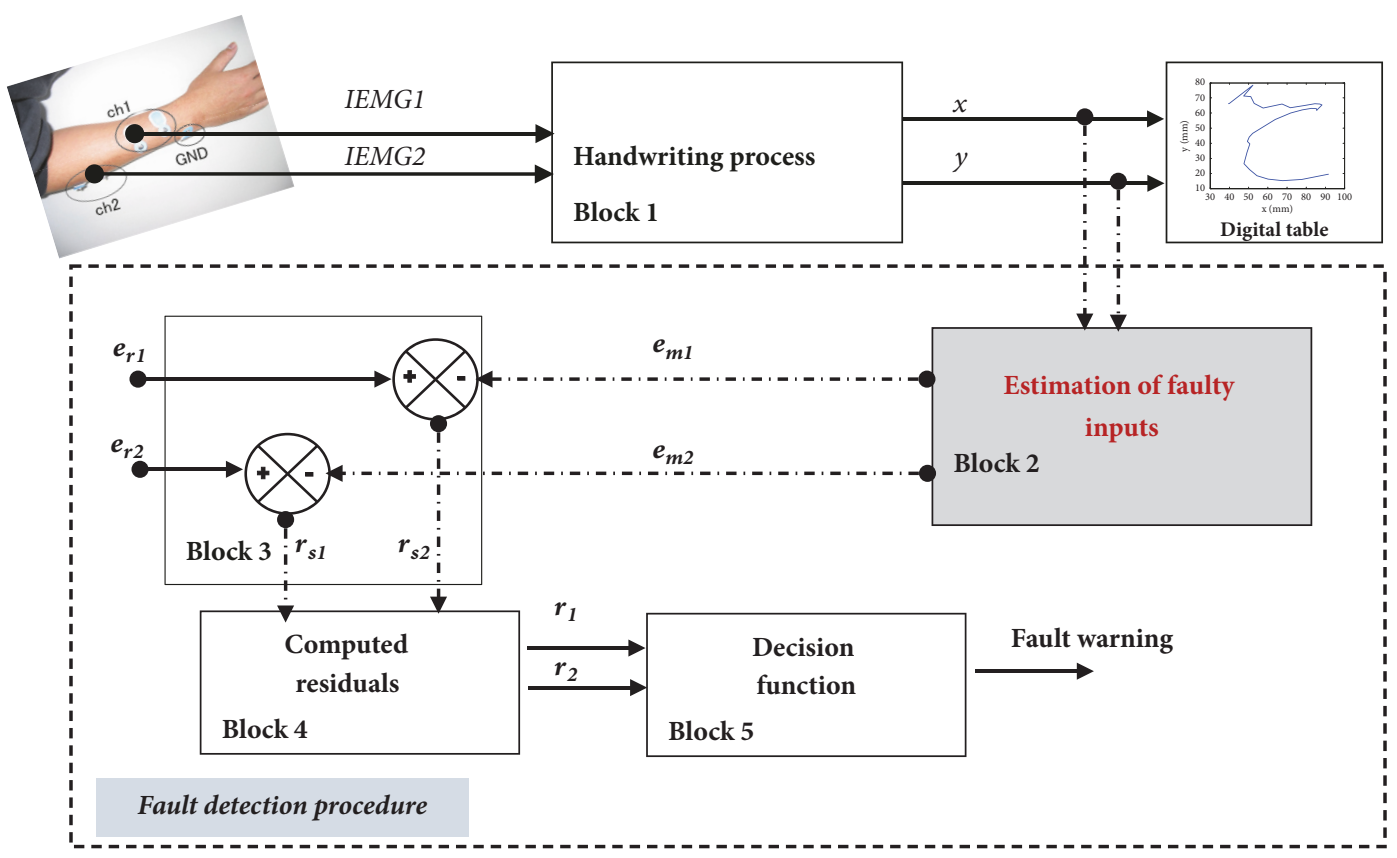

FIGURE 4: Synoptic schema of the proposed fault detection approach.

to the writers, the nature of the produced form, and even the variation of physical and psychical state of an individual. In summary, electromyographic signals are very sensitive to several distortions, so a fault detection approach is necessary to ensure a good functioning of the studied process.

In this sense, we propose the new fault detection approach introduced in Figure 4, based on predictive model using parametric identification model for the reconstruction of not healthy inputs (IEMG signals) of the handwriting assistance system using the writing coordinates on $(x, y)$ plane.

The proposed technique is applicable for several kinds of faults and can be useful in a lot of applications, such as hand prosthesis, smart electrodes maintenance, and military applications.

\section{Faults Study and Classification}

Before describing the proposed fault detection approach, we will present, in this section, the most frequent faults that can occur during the writing. Indeed, we can propose 3 main classes of faults [36-49]: technical, neurogenic, and modeling class.

(i) In the case of the technical class, faulty IEMG signal presents decrease/increase in the IEMG amplitude from a nominal one. We can cite, for example, muscles-electrodes 
distance, distance between electrodes and alignment to the fiber direction, displacement of electrodes from their initial position, and transmission cable.

(ii) In the case of neurogenic or myogenic class, related to neuronal and muscular problems, the faults lead to record a poor muscle activity signal with an increase of the IEMG signal amplitude from a nominal one, such as variation of the muscular temperature, morphologic variation, weariness of the muscles, and moving of electrodes during the writing act.

(iii) The case of modeling class is related to modeling problems, as variation of model parameters.

\section{Faulty Inputs Estimation from Outputs}

In this study, we are interested in studying faults of technical class. Knowing that most of these faults are related to IEMG signals, we choose to consider all distortions as actuator faults.

Figure 4 describes the studied approach that is based on a mathematical model (Block 1), developed by Chihi et al. [5], allowing us to mimic the handwriting motion. Otherwise, this model estimates coordinates of a drawing shape on the plane $(x, y)$ from IEMG signals. However, the performance of this model depends on the quality of IEMG signals, recorded by surface electrodes. As we already mentioned, these signals can present several faults and distortions.

The objective of the proposed fault detecting system is to detect and to localize a fault in the measured IEMG signals, inputs of the considered assistive handwriting system.

As it is presented in Figure 4, the main idea for detecting faults during this complex process is to propose a predicted model (Block 2) to estimate faulty inputs (IEMG signals) only from the coordinates of the written shape on the plane. The fault diagnostic algorithm is also based on the analysis of the absolute difference (Block 3) between the estimated $e_{m 1}$ and $e_{m 2}$, and the referential data, $e_{r 1}$ and $e_{r 2}$, of the forearm IEMG signals. These signals are recorded in good conditions without faults. A residue computing procedure is then proposed to define $r_{1}$ and $r_{2}$ (Block 4), which are used to decide and to generate fault warning (Block 5).

The residue, $r_{s i}, i=\{1,2\}$, is compared to a threshold value to detect and isolate the faults. Threshold is a parameter to be adjusted according the writer. In fact, each person presents his or her own reference IEMG signal, i.e., certain range of amplitude.

We note that $r_{s i}$ is the absolute difference between the faulty and the normal electromyography signal (5) and $r_{i}$ is the normalized residue (6).

$$
\begin{aligned}
& r_{s i}(k)=\mid\left(e_{r i}(k)-e_{m i}(k) \mid, \quad i=\{1,2\}\right. \\
& r_{i}(k)= \begin{cases}1, & \text { if } r_{s i}(k)>\text { threshold } \\
0, & \text { else }\end{cases}
\end{aligned}
$$

Figure 5 presents the flowchart of the proposed fault detection algorithm, based on the absolute residual computation which further allows defining a normalized residue to better present the instant of the fault appearance and its duration.

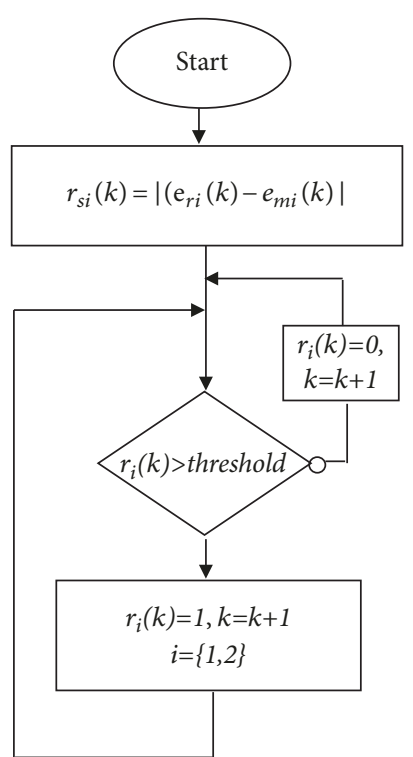

FIGURE 5: Flowchart of the fault detection and isolation procedure based on structural residue computations.

The proposed predicted model of faulty inputs (block 2 of Figure 4) is a MIMO fourth order model, whose inputs and outputs are interconnected. This model estimates both IEMG signals at the same time. It is based on velocities of the writing, $V_{x}$ and $V_{y}$, according to $x$ and $y$ coordinates, respectively. In fact, velocity of writing has inspired many researchers to solve various problems related to handwriting, such as the study of the rapid human movement [50], pattern recognition [51, 52], and even the handwriting modeling and control [53-55].

$$
\begin{aligned}
e_{m 1}(k)= & -\sum_{i=1}^{4}\left[a_{1 i} e_{m 2}(k-i)+b_{1 i} e_{m 1}(k-i)\right] \\
& +\sum_{i=1}^{4}\left[c_{1 i} V_{x}(k-i+1)+d_{1 i} V_{y}(k-i+1)\right] \\
e_{m 2}(k)= & -\sum_{i=1}^{4}\left[a_{2 i} e_{m 2}(k-i)+b_{2 i} e_{m 2}(k-i)\right] \\
& +\sum_{i=1}^{4}\left[c_{2 i} V_{x}(k-i+1)+d_{2 i} V_{y}(k-i+1)\right]
\end{aligned}
$$

where

$$
\begin{aligned}
& V_{x}, V_{y} \text { are the velocities of faulty coordinates, } x \text { and } y, \\
& \text { respectively, } \\
& e_{m 1}, e_{m 2} \text { are the estimated IEMG signals, } \\
& a_{1 i}, b_{1 i}, c_{1 i}, d_{1 i} \text { and } a_{2 i}, b_{2 i}, c_{2 i}, d_{2 i} \text { are the estimated } \\
& \text { parameters, relative to } e_{m 1} \text { and } e_{m 2} \text {, respectively. }
\end{aligned}
$$

The model parameters identification is based on classical Recursive Least Squares (RLS) algorithm with forgetting 


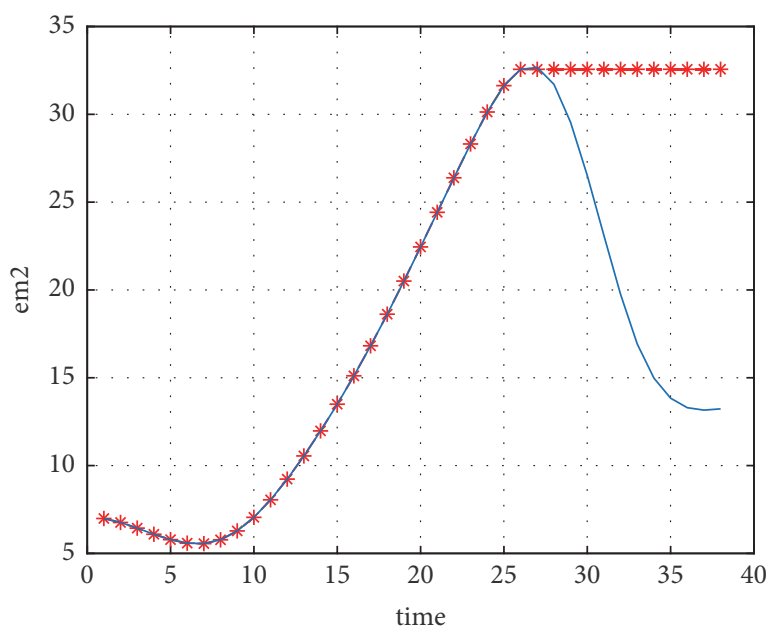

(a)
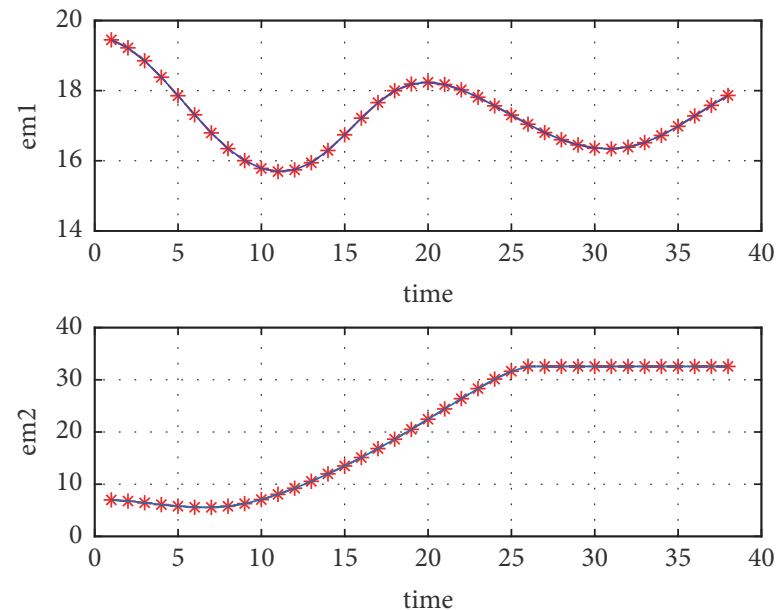

(b)

FIGURE 6: Step fault in IEMG signal. (a) Healthy and faulty IEMG signal. (b) Real and estimated faulty input.

factor equal to 0,95 which performs the following operations to update the parameters of the researched model [55-58].

$$
\begin{aligned}
& \widehat{\theta}(k)=\widehat{\theta}(k-1)+P(k) \sum_{i=n+1}^{k} y(i) \Psi(i) \\
& P(k)=P(k-1)-\frac{P(k-1) \Psi(k) \Psi^{T}(k) P(k-1)}{1+\Psi^{T}(k) P(k-1) \Psi(k)} \\
& \varepsilon(k)=y(k)-\hat{\theta}(k-1) \Psi(k)
\end{aligned}
$$

where

$$
\begin{aligned}
& \hat{\theta}(k) \text { is the vector of estimated parameters, } \\
& P(k) \text { is the adaptation matrix, } \\
& y(k) \text { is the actual output of the system to be identified, } \\
& \psi(k) \text { is the observation matrix, } \\
& \varepsilon(k) \text { is the estimated error. }
\end{aligned}
$$

The model structure used to identify the handwriting system dynamics for Multi-Inputs Multi-Outputs is also given as follows [55-58]:

$$
\begin{aligned}
& e_{m 1}=\psi_{1}^{T} \theta_{1}+\varepsilon_{1} \\
& e_{m 2}=\psi_{2}^{T} \theta_{2}+\varepsilon_{2}
\end{aligned}
$$

where

$\varepsilon_{1}$ and $\varepsilon_{2}$ are the error vectors, relative to $e_{m 1}$ and $e_{m 2}$ signals, respectively,

$\psi_{1}^{\mathrm{T}}$ and $\psi_{2}^{\mathrm{T}}$ are the matrices whose elements are relative to $e_{m 1}$ and $e_{m 2}$ signals, respectively.

4.1. Simulation Results. In order to validate the proposed fault detection strategy, several simulations were performed in Matlab/Simulink with real measurement files of EMG signals and its corresponding pen tip movement coordinates.
We note that $e_{d}$ is the faulty input signal, which summarizes the frequent technical faults that could attempt the handwriting process and presents the model of each one [3649].

4.1.1. Case 1: One Faulty Input. In a first analysis, we consider that one fault can affect only one IEMG signal, measured using surface electrodes. Therefore, we propose studying these kinds of faults: step, decrease of amplitude, ramp, random, and intermittent.

For Figures 6-8, we note that the estimated IEMG signals are presented in red line with stars and real signals are in continuous blue line. Curves (a) present IEMG signal without fault (blue continuous line) and the faulty input (red line with stars). Curves (b) show the real (blue continuous line) and the estimated faulty inputs (red line with stars) using model (3).

(i) Step Fault. Step fault, expressed by (9), is mainly caused by the distortion of electrodes that can stay blocked by generating a constant amplitude, Figure 6 .

$$
e_{d}\left(t_{k}\right)= \begin{cases}e & \text { if } t_{k}<t_{f} \\ f & \text { else } t_{k} \geq t_{f}\end{cases}
$$

$f$ is constant,

$t_{k}$ is the discreet instant,

$t_{f}$ is the instant of occurrence of the fault,

$e$ is the not healthy input.

Figure 6(a) shows the difference between the estimated signal (represented by red stars curves) and its corresponding referential data (represented by continuous blue curves). Figure 6(b) presents the ability of the proposed approach to predict IEMG signal, despite the abrupt changing of the studied signal. 


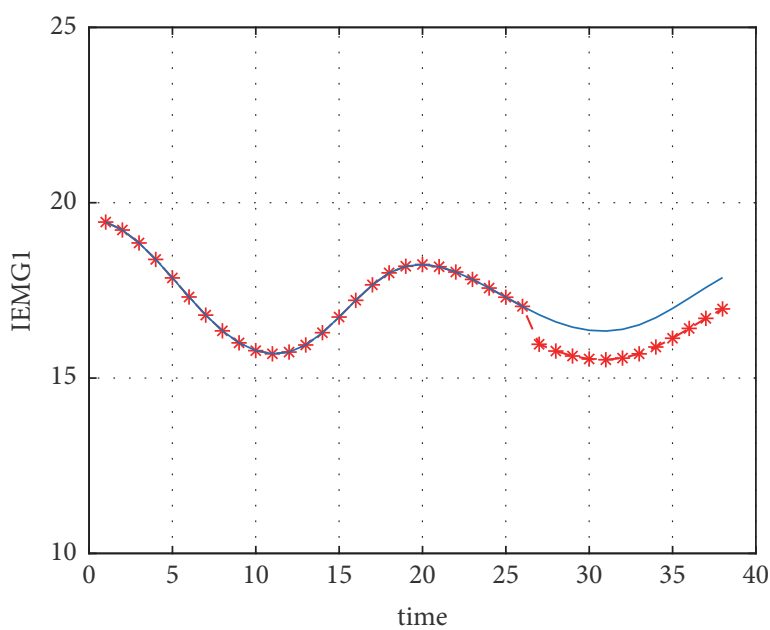

(a)
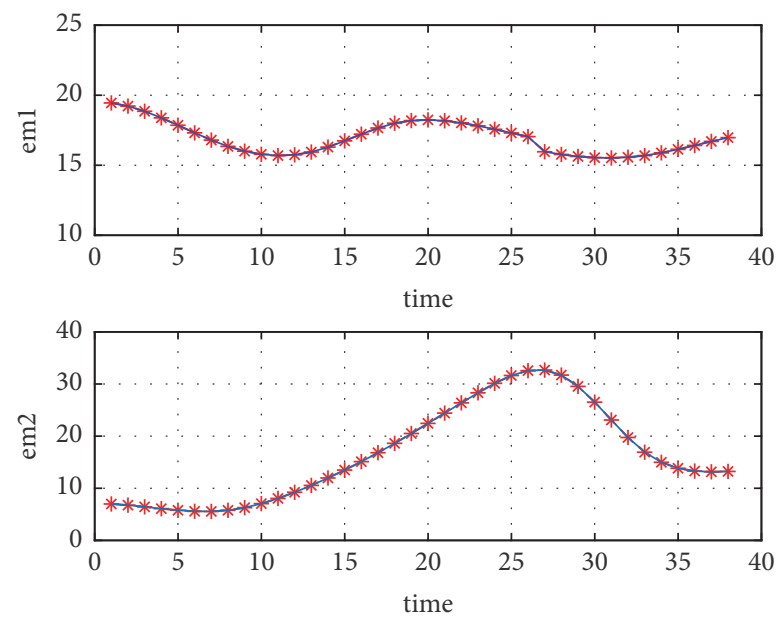

(b)

FIGURE 7: Decrease of the amplitude fault in IEMG signal. (a) Healthy and faulty IEMG signal. (b) Real and estimated faulty input.
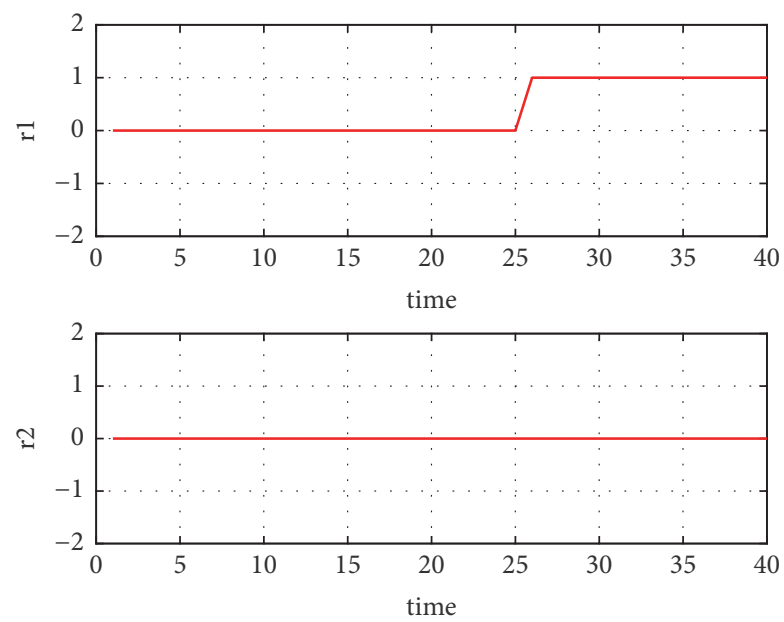

FIGURE 8: Normalized residue for step and decrease of the amplitude faults.

(ii) Decrease of the Amplitude Fault. In this study, we take into consideration the disadvantages of most myoelectric prostheses, which are summed up in the variation of the distances between electrodes or between muscles-electrodes, the alignment to the fiber direction, etc.

The impact of these faults on IEMG signals can be represented by a decrease of the amplitude, Figure 7.

This type of distortion can cause problems in the control and precision of the prosthesis, which is very embarrassing for the user.

The normalized residue $r_{i}$ is equal to one during the fault occurrence. Figure 8 presents the residue relative to the cases step and decrease of the amplitude faults.

(iii) Ramp Fault. The distortion due to the aging of the electronic components can be represented by a ramp function
(10). Figure 9 shows good estimation of this type of fault using the proposed approach.

$$
e_{d}\left(t_{k}\right)=\left\{\begin{array}{ll}
e & \text { if } t_{k}<t_{f} \\
\alpha f & \text { else } t_{k} \geq t_{f}
\end{array} \quad \alpha=\right.\text { constant }
$$

(iv) Random Fault. The faulty input is also estimated with the presence of a random distortion which can be caused by a technical problem in the transmission cable, or an unknown external interference, etc. Generally random fault is difficult to estimate; however the proposed approach shows a good concordance between the real and the estimated faulty signal, Figure 10. The normalized residue is illustrated by Figure 11 .

(v) Intermittent Fault. The estimation of inputs with an intermittent fault (11) is also taken into account. In effect, micro cuts, instantaneous displacement of electrodes, or even unpredictable changes in IEMG signals can be considered as an intermittent fault (ramp, step, random, etc.).

$$
e_{d}= \begin{cases}f_{\text {int }}\left(t_{k}\right), & t_{f 1} \leq t_{k} \leq t_{f 2} \\
e, & t_{k} \notin\left[\begin{array}{ll}
t_{f 1} & t_{f 2}
\end{array}\right]\end{cases}
$$

$t_{f 1}$ and $t_{f 2}$ are instants of the beginning and the end of the fault.

Figure 12 shows good concordance between the real inputs and the estimated signals. The normalized residue is equal to one during the fault occurrence, Figure 13.

4.1.2. Case 2: Tow Faulty Inputs. In a second analysis, we suppose that both inputs are sensitive to two successive faults.

In order to show the relevance of the proposed approach, we start with the case where both IEMG signals have only one fault, Figure 10(a). Figure 10(b) presents the estimated and the real not healthy signals in the case of two faults in each electromyographic signal. 


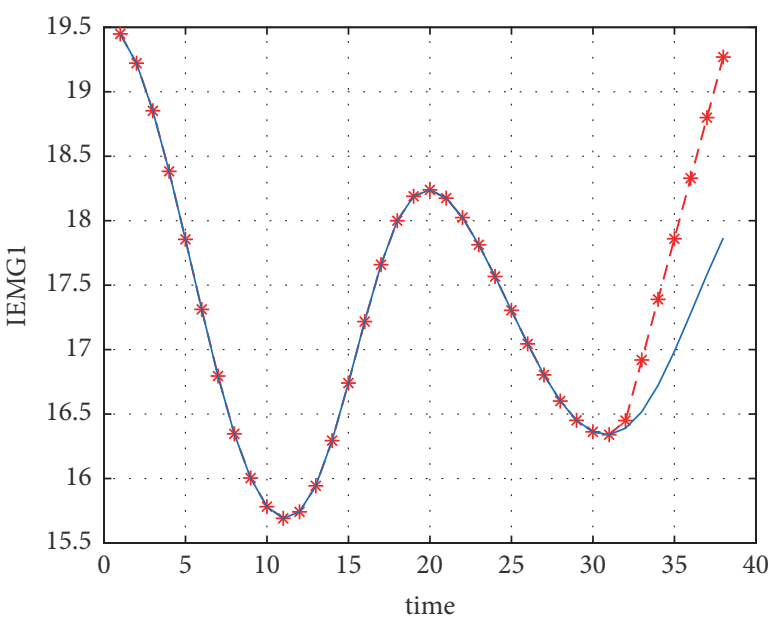

(a)
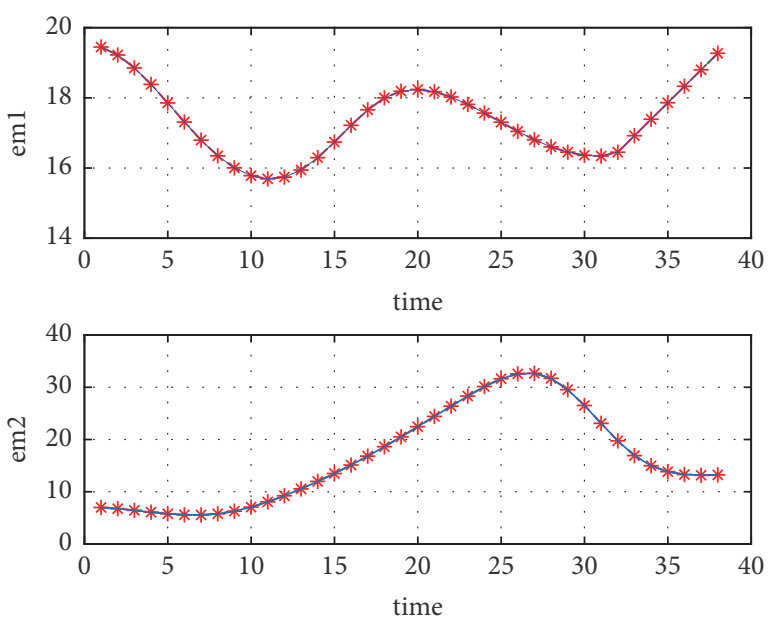

(b)

FIGURE 9: Ramp fault in IEMG signal. (a) Healthy and faulty IEMG signal. (b) Real and estimated faulty input.

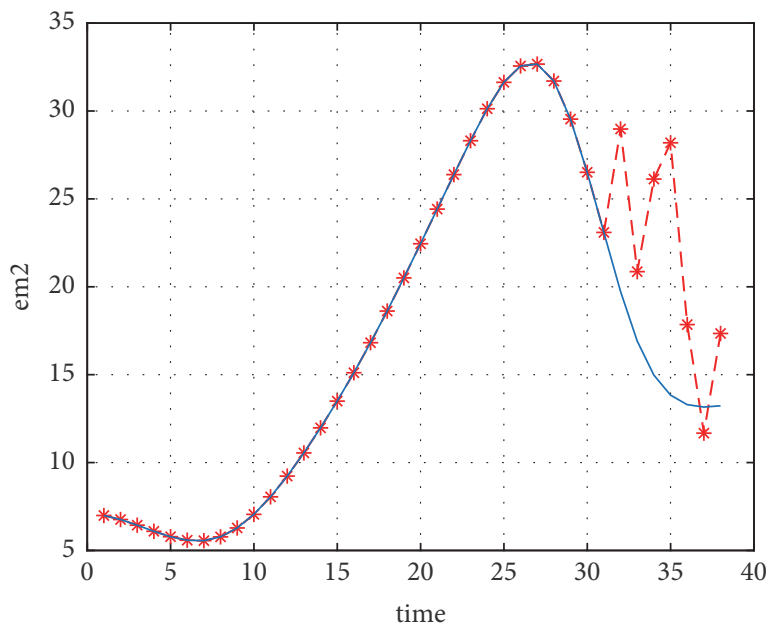

(a)
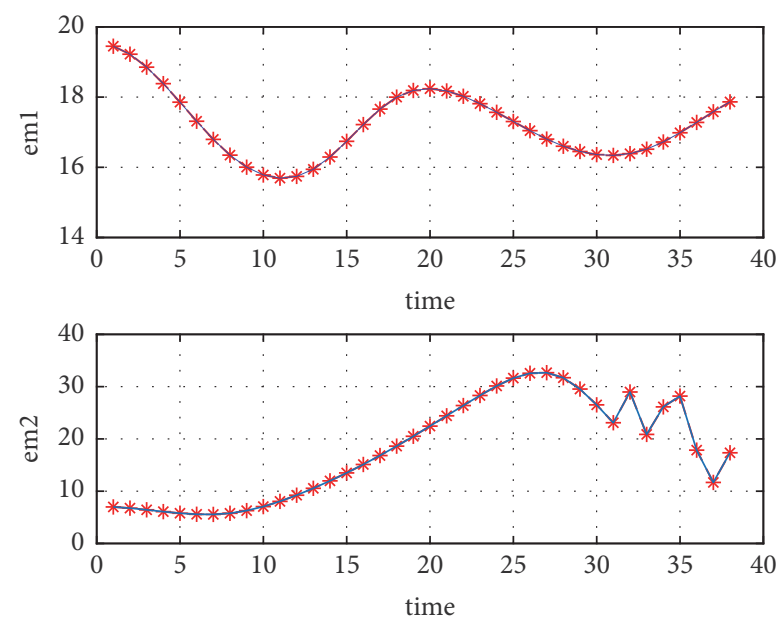

(b)

FIGURE 10: Step fault in IEMG2 signal. (a) Healthy and faulty IEMG signal. (b) Real and estimated faulty input.
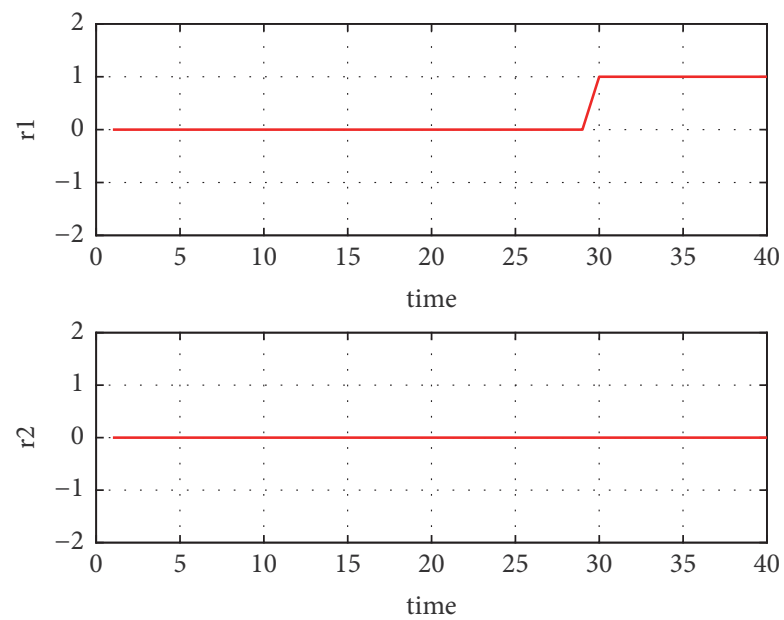

FIGURE 11: Normalized residue for step and decrease of the amplitude faults.
The normalized residues give information on the time of occurrence of the fault and its duration. Residues of each input are different to zero in the presence of any kind of fault, Figures $14(\mathrm{a})^{\prime}$ and $14(\mathrm{~b})^{\prime}$.

To evaluate the proposed fault detection approach, one or more IEMG signals in the testing data set were artificially distorted. Therefore, step, ramp, intermittent, and random faults were adjusted to simulate the different disturbances commonly occurring at EMG signal. The proposed approach shows good concordance to estimate faulty recorded muscles activities from damaged shapes coordinates on the plane.

In order to make handwriting practical and available to people with motor deficits, real writing challenges resulting in deformation in EMG signals must be overcome. This work aims to address these challenges by proposing a fault detection approach to mimic the behavior of individual distorted EMG signals. 


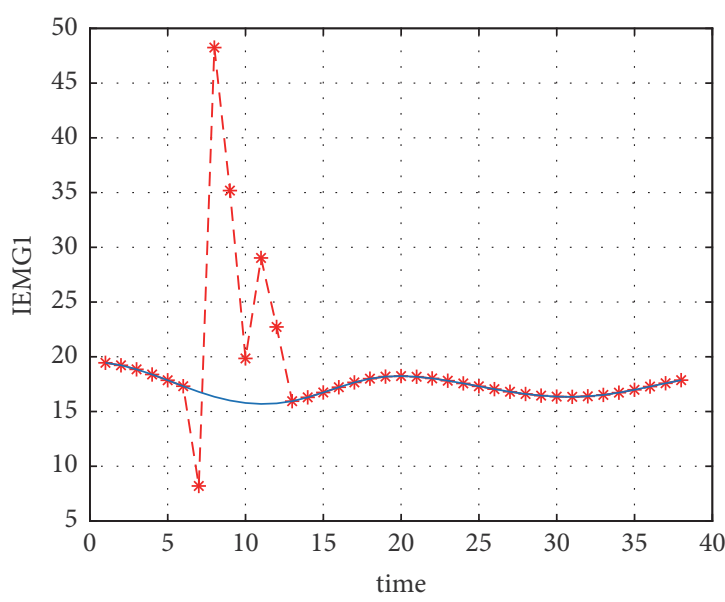

(a)
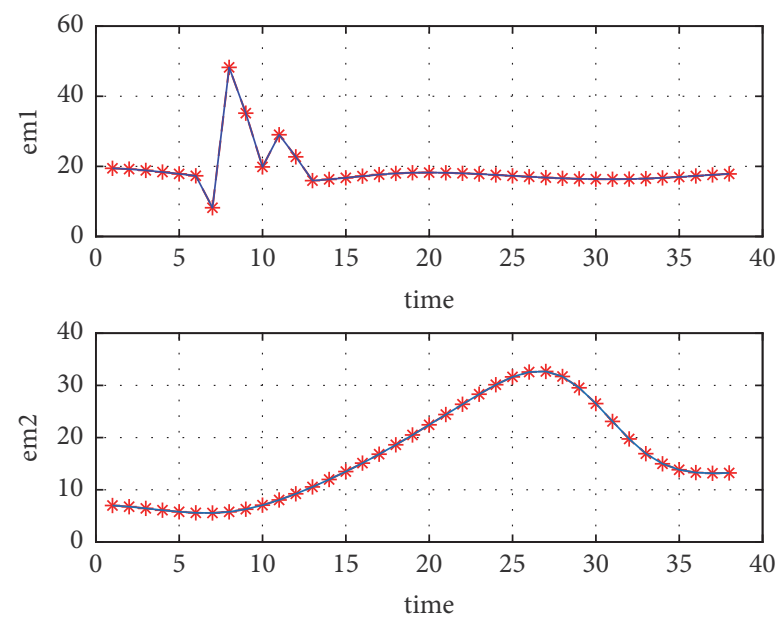

(b)

FIGURE 12: Random fault in IEMG1 signal. (a) Healthy and faulty IEMG1 signal. (b) Real and estimated faulty input.
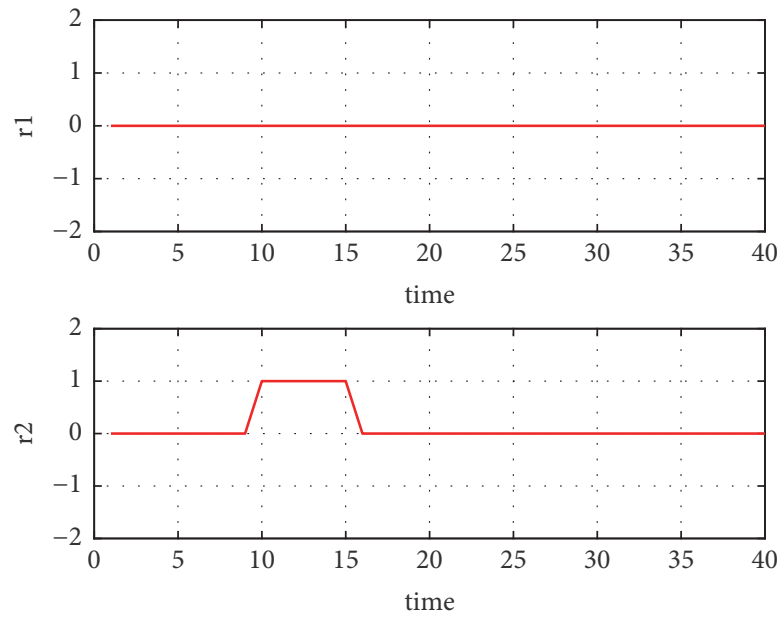

FIGURE 13: Normalized residue for intermittent fault in IEMG2 signal.

The results of this study demonstrate several benefits of the designed fault detection approach, such as the adequacy to correct for sudden signal disturbances simulated in the present study and the possibility of detecting one or multiple distorted EMG signals.

The proposed fault detection approach is considered as an alternative solution to the unknown input observer based techniques allowing model-independent fault detection without any observability requirements.

\section{Conclusion}

The present work developed a new model-free fault detection technique for unpredictable biological inputs. For this, we presented a classification study of the different kinds of faults that can affect the writing process. Then parametric identification model with RLS algorithm was proposed to reconstruct faulty integrated EMG signals. Thereafter, from the fault reconstruction results, a normalized residual is computed to facilitate the fault detection procedure from damaged system outputs.

The proposed fault detection method showed high performance in detecting faults of different types and for one or multiple distorted EMG signals.

These promising outcomes could inform the design of clinically viable EMG estimation and eventually benefit individuals with motor deficits.

\section{Data Availability}

The [EMG signals and the corresponding letters coordinates] data used to support the findings of this study are available from the corresponding author upon request. 

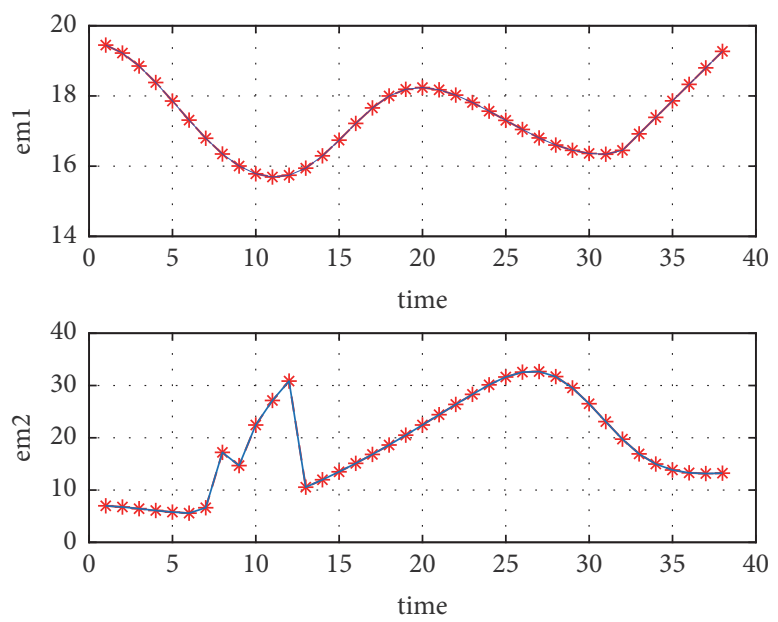

(a)
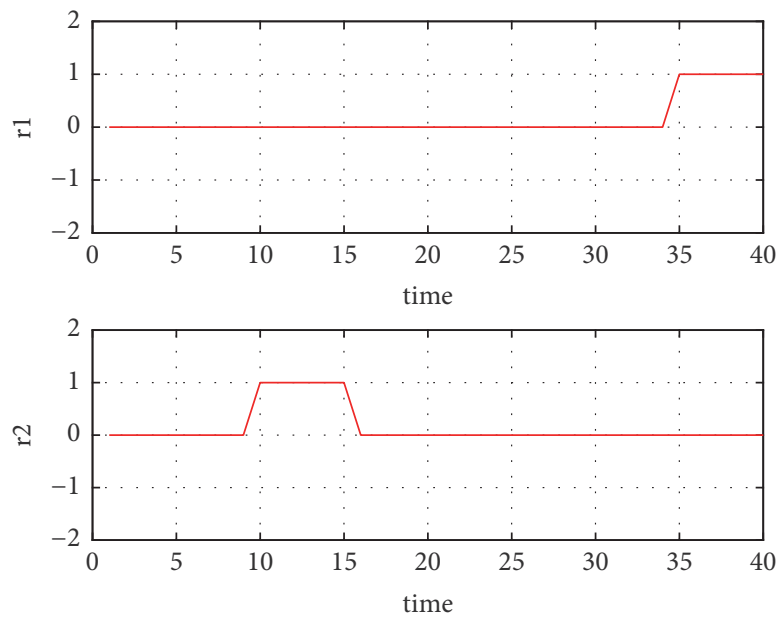

(a) ${ }^{\prime}$
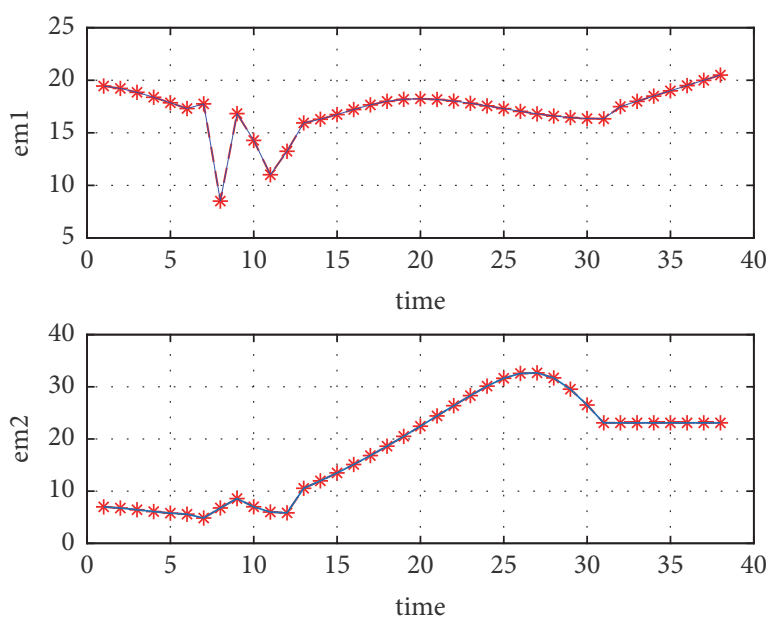

(b)
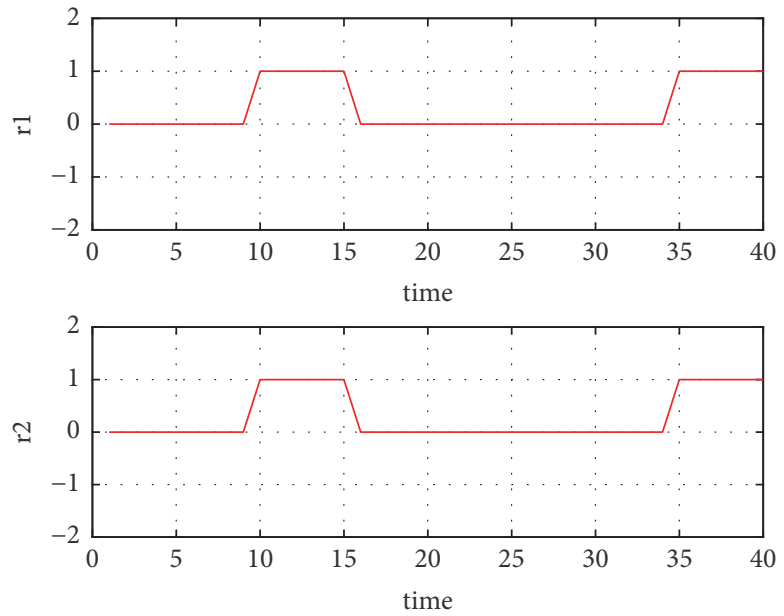

(b)

Figure 14: Two faulty IEMG signals (a) One fault in each signal, (b) Two faults in each signal (a) $)^{\prime}$ Normalized residue of (a), (b) ${ }^{\prime}$ Normalized residue of (b).

\section{Conflicts of Interest}

The authors declare that there are no conflicts of interest regarding the publication of this paper.

\section{References}

[1] I. Kuzborskij, A. Gijsberts, and B. Caputo, "On the challenge of classifying 52 hand movements from surface electromyography," in Proceedings of the 34th Annual International Conference of the IEEE Engineering in Medicine and Biology Society, EMBS 2012, pp. 4931-4937, USA, September 2012.

[2] J. G. Hincapie and R. F. Kirsch, "Feasibility of EMG-based neural network controller for an upper extremity neuroprosthesis," IEEE Transactions on Neural Systems and Rehabilitation Engineering, vol. 17, no. 1, pp. 80-90, 2009.

[3] L. Rossini and P. M. Rossini, "Combining ENG and EEG integrated analysis for better sensitivity and specificity of neuroprosthesis operations," in Proceedings of the 2010 32nd Annual International Conference of the IEEE Engineering in Medicine and Biology Society, EMBC'10, pp. 134-137, Argentina, September 2010.
[4] T. R. D. Scott and V. A. Vare, "A novel five degree of freedom user command controller in people with spinal cord injury and non-injured for full upper extremity neuroprostheses, wearable powered orthoses and prosthetics," Medical \& Biological Engineering \& Computing, vol. 51, no. 3, pp. 317-330, 2013.

[5] I. Chihi, A. Abdelkrim, and M. Benrejeb, "Multi-model approach to characterize human handwriting motion," Biological Cybernetics, vol. 110, no. 1, pp. 17-30, 2016.

[6] M. Badri, I. Chihi, L. Sidhom, and A. Abdelkrim, "Observer approach for fault detection of the handwriting process," in Proceedings of the 4th International Conference on Control Engineering and Information Technology, CEIT 2016, Tunisia, December 2016.

[7] S. Bitzer and P. Van Der Smagt, "Learning EMG control of a robotic hand: towards active prostheses," in Proceedings of the IEEE International Conference on Robotics and Automation (ICRA '06), pp. 2819-2823, IEEE, May 2006.

[8] S. Schulz, C. Pylatiuk, M. Reischl, J. Martin, R. Mikut, and G. Bretthauer, "A hydraulically driven multifunctional prosthetic hand," Robotica, vol. 23, no. 3, pp. 293-299, 2005. 
[9] M. Zecca, S. Micera, M. C. Carrozza, and P. Dario, "Control of multifunctional prosthetic hands by processing the electromyographic signal," Critical Reviews in Biomedical Engineering, vol. 30, no. 4-6, pp. 459-485, 2002.

[10] R. N. Scott and P. A. Parker, "Myoelectric prostheses: State of the art," Journal of Medical Engineering \& Technology, vol. 12, no. 4, pp. 143-151, 1988.

[11] R. B. Jerard, T. W. Williams, and C. W. Ohlenbusch, "Practical Design of an EMG Controlled Above Elbow Prosthesis, Conference on Engineering of Devices for Rehabilitation," in Conference on Engineering of Devices for Rehabilitation, p. 73, Tufts University School of Medicine, 1974.

[12] S. C. Jacobsen, D. F. Knutti, R. T. Johnson, and H. H. Sears, "Development of the Utah Artificial Arm," IEEE Transactions on Biomedical Engineering, vol. 29, no. 4, pp. 249-269, 1982.

[13] P. K. Artemiadis and K. J. Kyriakopoulos, "An EMG-based robot control scheme robust to time-varying EMG signal features," IEEE Transactions on Information Technology in Biomedicine, vol. 14, no. 3, pp. 582-588, 2010.

[14] P. K. Artemiadis and K. J. Kyriakopoulos, "A switching regime model for the emg-based control of a robot arm," IEEE Transactions on Systems, Man, and Cybernetics, Part B: Cybernetics, vol. 41, no. 1, pp. 53-63, 2011.

[15] P. K. Artemiadis and K. J. Kyriakopoulos, "Assessment of muscle fatigue using a probabilistic framework for an EMGbased robot control scenario," in Proceedings of the 8th IEEE International Conference on BioInformatics and BioEngineering, BIBE 2008, Greece, October 2008.

[16] P. Parker, K. Englehart, and B. Hudgins, "Myoelectric signal processing for control of powered limb prostheses," Journal of Electromyography \& Kinesiology, vol. 16, no. 6, pp. 541-548, 2006.

[17] L. J. Hargrove, K. Englehart, and B. Hudgins, "A comparison of surface and intramuscular myoelectric signal classification," IEEE Transactions on Biomedical Engineering, vol. 54, no. 5, pp. 847-853, 2007.

[18] J. W. Sensinger, B. A. Lock, and T. A. Kuiken, "Adaptive pattern recognition of myoelectric signals: Exploration of conceptual framework and practical algorithms," IEEE Transactions on Neural Systems and Rehabilitation Engineering, vol. 17, no. 3, pp. 270-278, 2009.

[19] C. J. De Luca, L. D. Gilmore, M. Kuznetsov, and S. H. Roy, "Filtering the surface EMG signal: movement artifact and baseline noise contamination," Journal of Biomechanics, vol. 43, no. 8, pp. 1573-1579, 2010.

[20] R. H. Chowdhury, M. B. Reaz, M. A. B. M. Ali, A. A. Bakar, K. Chellappan, and T. Chang, "Surface electromyography signal processing and classification techniques," Sensors, vol. 13, no. 9, pp. 12431-12466, 2013.

[21] K. de Moura and A. Balbinot, "Virtual Sensor of Surface Electromyography in a New Extensive Fault-Tolerant Classification System," Sensors, vol. 18, no. 5, p. 1388, 2018.

[22] X. Zhang and H. Huang, "A real-time, practical sensor faulttolerant module for robust EMG pattern recognition," Journal of NeuroEngineering and Rehabilitation, vol. 12, no. 1, pp. 12-18, 2015.

[23] S. Das Gupta, "On The Generalized Distance in Statistics," in Proceedings of the National Institute of Sciences:Calcutta, vol. 2, pp. 49-55, 1936.

[24] L. Resnik, H. H. Huang, A. Winslow, D. L. Crouch, F. Zhang, and N. Wolk, "Evaluation of EMG pattern recognition for upper limb prosthesis control: A case study in comparison with direct myoelectric control," Journal of NeuroEngineering and Rehabilitation, vol. 15, no. 1, 2018.

[25] H. Huang, F. Zhang, Y. L. Sun, and H. He, "Design of a robust EMG sensing interface for pattern classification," Journal of Neural Engineering, vol. 7, no. 5, Article ID 56005, 2010.

[26] Y. Zhang and J. Jiang, "Bibliographical review on reconfigurable fault-tolerant control systems," Annual Reviews in Control, vol. 32, no. 2, pp. 229-252, 2008.

[27] L. Sidhom and I. Chihi, "Fault actuators detection based on inputs-outputs data mapping: application to a robot manipulator," International Journal of Advanced Mechatronic Systems, vol. 7, no. 5, pp. 328-336, 2017.

[28] J. Gertler, Fault Detection and Diagnosis in Engineering Systems, Marcel Dekker, New York, NY, USA, 1998.

[29] N. Briquet-Kerestedjian, M. Makarov, M. Grossard, and P. Rodriguez-Ayerbe, "Stochastic observer design for robot impact detection based on inverse dynamic model under uncertainties," in 20th World Congress of the Inter. Federation of Automatic Control, Toulouse, France, August 2017.

[30] A. S. Rezazadeh, H. R. Koofigar, and S. Hosseinnia, "Adaptive fault detection and isolation for a class of robot manipulators with time-varying perturbation," Journal of Mechanical Science and Technology, vol. 29, no. 11, pp. 4901-4911, 2015.

[31] R. Patton, P. Frank, and R. Clark, Issues of Fault Diagnosis for Dynamic Systems, Springer, London, UK, 2000.

[32] H. Liu and G. M. Coghill, "A model-based approach to robot fault diagnosis," Knowledge-Based Systems, vol. 18, no. 4-5, pp. 225-233, 2005.

[33] H. A. Talebi, K. Khorasani, and S. Tafazoli, "A recurrent neuralnetwork-based sensor and actuator fault detection and isolation for nonlinear systems with application to the satellite's attitude control subsystem," IEEE Transactions on Neural Networks and Learning Systems, vol. 20, no. 1, pp. 45-60, 2009.

[34] I. Eski, S. Erkaya, S. Savas, and S. Yildirim, "Fault detection on robot manipulators using artificial neural networks," Robotics and Computer-Integrated Manufacturing, vol. 27, no. 1, pp. 115123, 2011.

[35] M. Sano, T. Kosaku, and Y. Murata, Modeling of Human Handwriting Motion by Electromyographic Signals on Forearm Muscles, CCCT'03, Orlando, Fl, USA, 2003.

[36] S. X. Ding, Model-Based Fault Diagnosis Techniques, SpringerVerlag, Berlin, Germany, 2008.

[37] Z. Gao, C. Cecati, and S. X. Ding, "A survey of fault diagnosis and fault-tolerant techniques-part I: fault diagnosis with model-based and signal-based approaches," IEEE Transactions on Industrial Electronics, vol. 62, no. 6, pp. 3757-3767, 2015.

[38] J. H. Fan, Y. M. Zhang, and Z. Q. Zheng, "Adaptive observerbased integrated fault diagnosis and fault-tolerant control systems against actuator faults and saturation," Journal of Dynamic Systems, Measurement, and Control, vol. 135, no. 4, Article ID 041008, 13 pages, 2013.

[39] M. Witczak, J. Korbicz, and R. J'Ozefowicz, "Design of unknown input observers for non-linear stochastic systems and their application to robust fault diagnosis," Control and Cybernetics, vol. 42, no. 1, pp. 227-256, 2013.

[40] V. Reppa and A. Tzes, "Fault diagnosis based on set membership identification using output-error models," International Journal of Adaptive Control and Signal Processing, vol. 30, no. 2, pp. 224$255,2015$. 
[41] L. H. LindstrÖM and R. I. Magnusson, "Interpretation of Myoelectric Power Spectra: A Model and Its Applications," Proceedings of the IEEE, vol. 65, no. 5, pp. 653-662, 1977.

[42] S. C. Gandevia and D. K. McKenzie, "Human diaphragmatic EMG: Changes with lung volume and posture during supramaximal phrenic stimulation," Journal of Applied Physiology, vol. 60, no. 4, pp. 1420-1428, 1986.

[43] A. E. Grassino, W. A. Whitelaw, and J. Milic Emili, "Influence of lung volume and electrode position on electromyography of the diaphragm," Journal of Applied Physiology, vol. 40, no. 6, pp. 971-975, 1976.

[44] E. Onal, M. Lopata, A. S. Ginzburg, and T. D. O'Connor, "Diaphragmatic EMG and transdiaphragmatic pressure measurements with a single catheter," The American Review of Respiratory Disease, vol. 124, pp. 563-568, 1981.

[45] J. Beck, C. Sinderby, J. Weinberg, and A. Grassino, "Effects of muscle-to-electrode distance on the human diaphragm electromyogram," Journal of Applied Physiology, vol. 79, no. 3, pp. 975-985, 1995.

[46] J. A. Daubenspeck, J. C. Leiter, J. F. McGovern, S. L. Knuth, and E. J. Kobylarz, "Diaphragmatic electromyography using a multiple electrode array," Journal of Applied Physiology, vol. 67, no. 4, pp. 1525-1534, 1989.

[47] C. A. Sinderby, A. S. Comtois, R. G. Thomson, and A. E. Grassino, "Influence of the bipolar electrode transfer function on the electromyogram power spectrum," Muscle \& Nerve, vol. 19, no. 3, pp. 290-301, 1996.

[48] D. V. Roberts, "Simultaneous measurement of propagation velocity of action potentials and contraction wave in frog striated muscle," The Journal of Physiology, vol. 147, pp. 62-68, 1969.

[49] R. Fink and H. C. Lüttgau, "An evaluation of the membrane constants and the potassium conductance in metabolically exhausted muscle fibres.," The Journal of Physiology, vol. 263, no. 2, pp. 215-238, 1976.

[50] R. Plamondon, A. M. Alimi, P. Yergeau, and F. Leclerc, "Modelling velocity profiles of rapid movements: a comparative study," Biological Cybernetics, vol. 69, no. 2, pp. 119-128, 1993.

[51] A. Fischer and R. Plamondon, "Signature Verification Based on the Kinematic Theory of Rapid Human Movements," IEEE Transactions on Human-Machine Systems, vol. 47, no. 2, pp. 169180, 2017.

[52] H. Boubaker, A. Chaabouni, N. Tagougui, M. Kherallah, and A. M. Alimi, "Handwriting and Hand Drawing Velocity Modeling by Superposing Beta Impulses and Continuous Training Component," International Journal of Computer Science Issues, vol. 10, no. 5, 2013.

[53] M. Benrejeb and A. El Abed-Abdelkrim, "Speed analysis of handwriting process models," in Proceedings of the SMC2002: IEEE International Conference on Systems, Man and Cybernetics, pp. 276-281, Yasmine Hammamet, Tunisia, 2002.

[54] I. Chihi, A. Abdelkrim, and M. Benrejeb, "Analysis of handwriting velocity to identify handwriting process from Electromyographic signals," American Journal of Applied Sciences, vol. 9, no. 10, pp. 1742-1756, 2012.

[55] M. A. Slim, A. Abdelkrim, and M. Benrejeb, "Handwriting velocity modeling by sigmoid neural networks with Bayesian regularization," in Proceedings of the 2014 International Conference on Electrical Sciences and Technologies in Maghreb, CISTEM 2014, pp. 1-7, Tunisia, November 2014.

[56] A. Vahidi, A. Stefanopoulou, and H. Peng, "Recursive least squares with forgetting for online estimation of vehicle mass and road grade: theory and experiments," Vehicle System Dynamics, vol. 43, no. 1, pp. 31-55, 2005.

[57] T. Tutunji, M. Molhim, and E. Turki, "Mechatronic systems identification using an impulse response recursive algorithm," Simulation Modelling Practice and Theory, vol. 15, no. 8, pp. 970 988, 2007.

[58] T. Jayaprabha and A. Suresh, "Blood flow, vein and nerves detector using an NIR sensor with RLS estimation for embedded signal processing," in Proceedings of the 2016 International Conference on Advanced Communication Control and Computing Technologies, ICACCCT 2016, pp. 117-121, India, May 2016. 


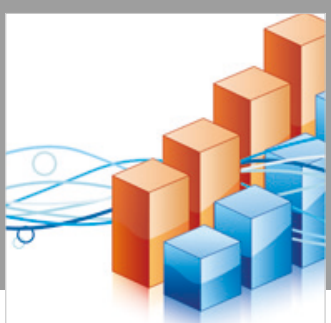

Advances in

Operations Research

\section{-n-m}
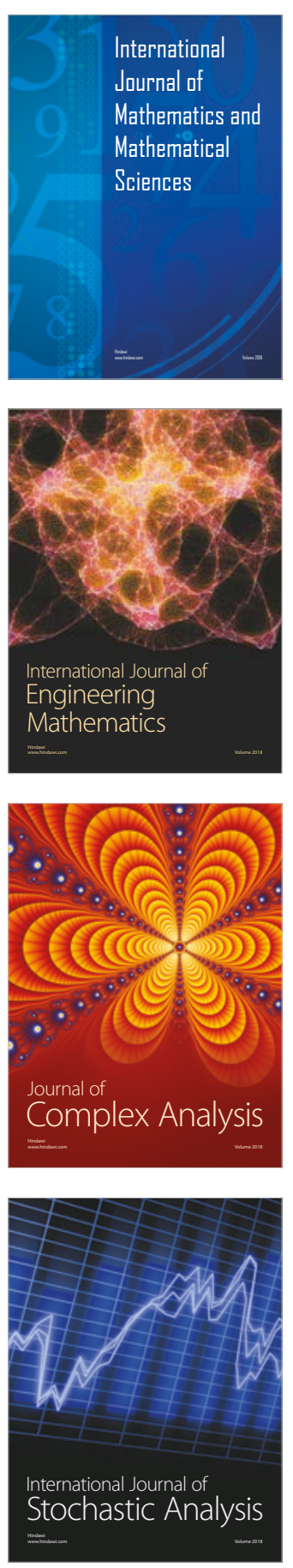
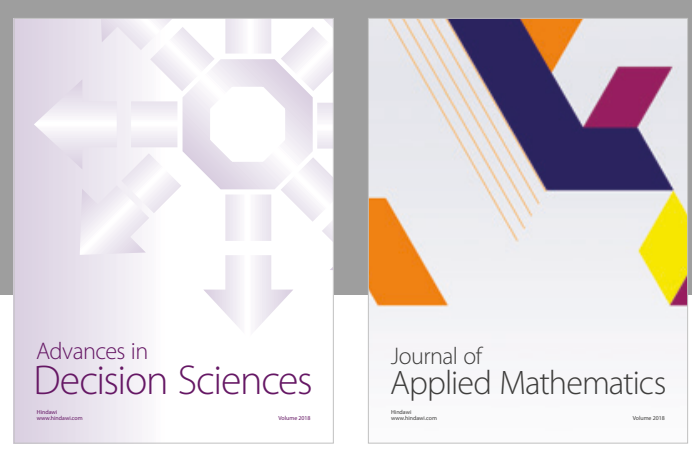

Journal of

Applied Mathematics
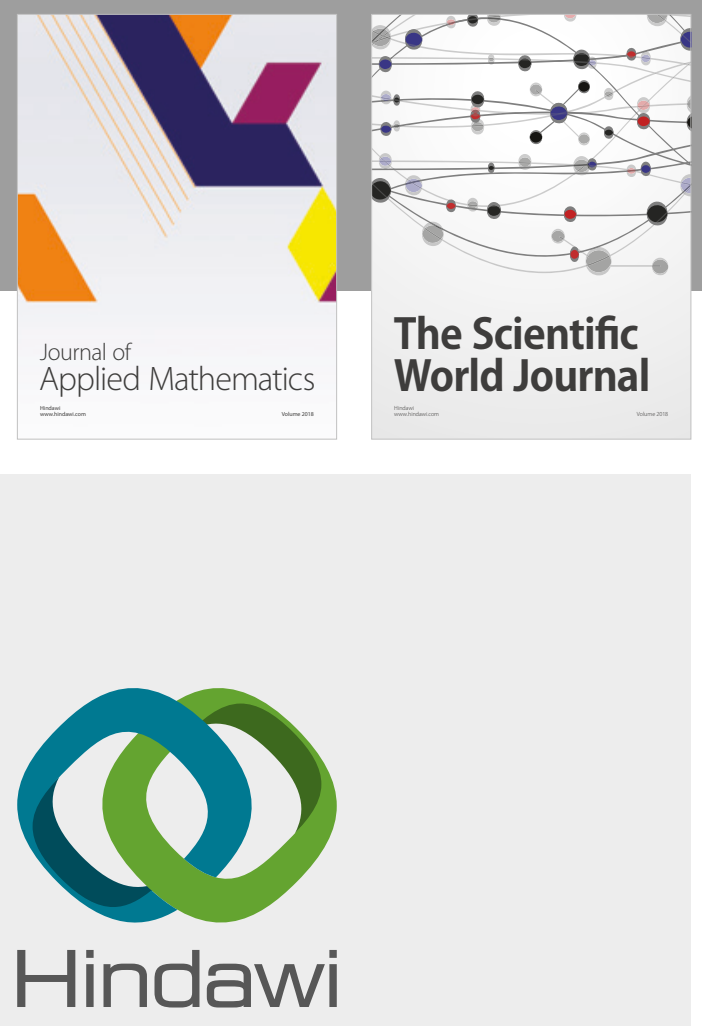

Submit your manuscripts at

www.hindawi.com

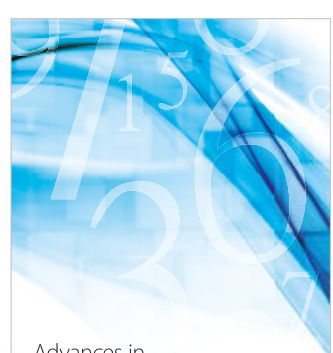

Advances in
Numerical Analysis
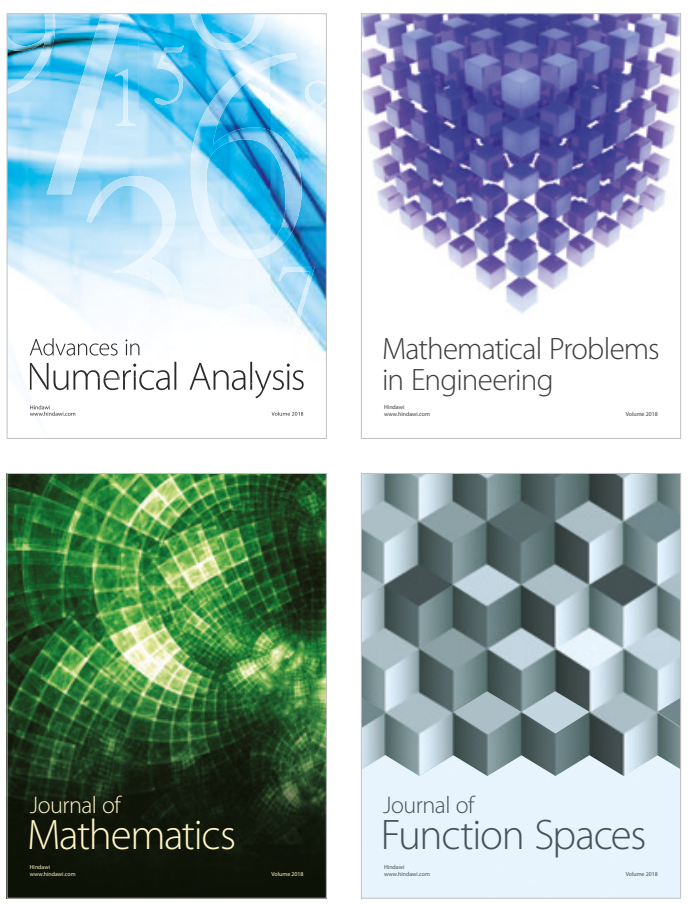

Mathematical Problems in Engineering

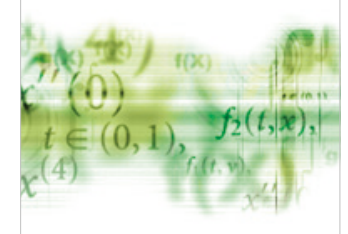

International Journal of

Differential Equations

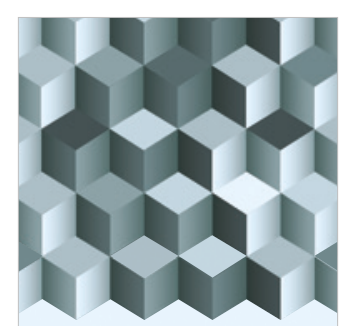

Journal of

Function Spaces
The Scientific

World Journal

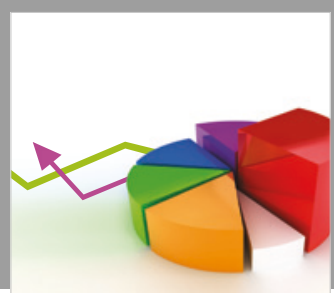

Journal of

Probability and Statistics
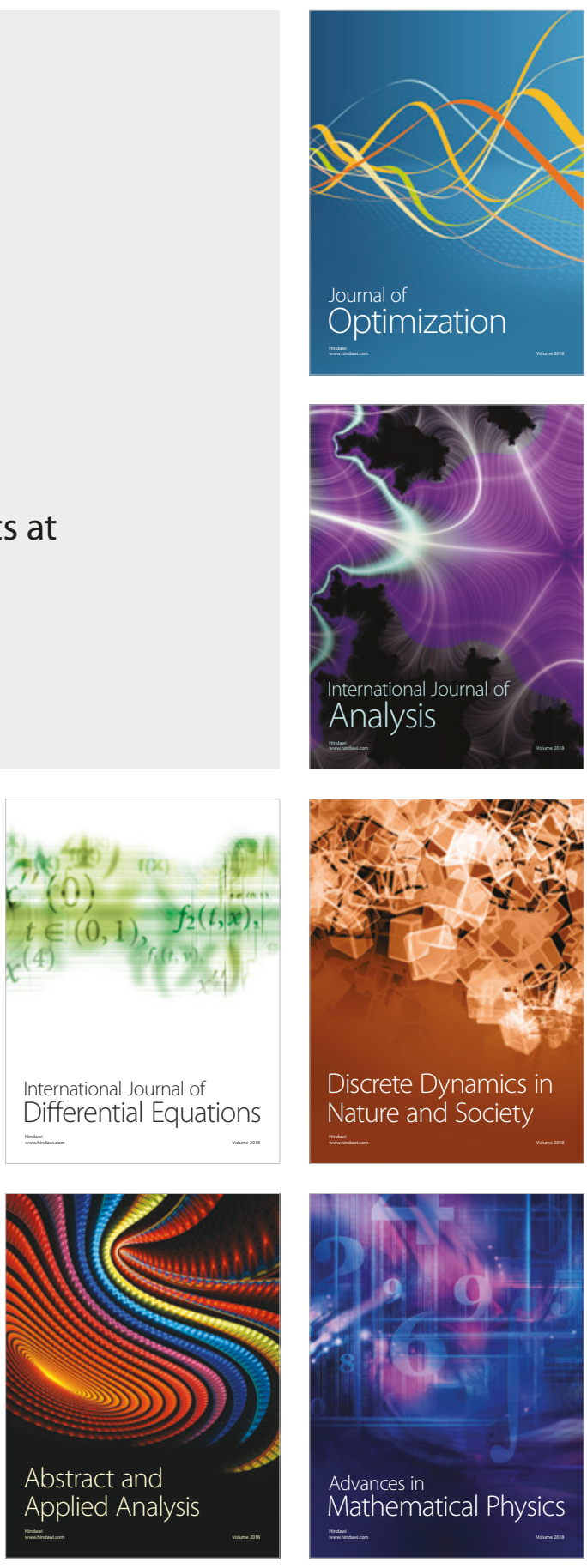